\title{
The Alcubierre Warp Drive in Higher Dimensional Spacetime
}

\author{
H. G. White ${ }^{1}$ and E. W. Davis ${ }^{2}$ \\ ${ }^{1} 5231$ Pilgrim Oaks Lane, League City, TX 77573, USA \\ ${ }^{2}$ Inst. for Advanced Studies at Austin, 4030 W. Braker Ln., Ste. 300, Austin, TX 78759, USA \\ '281-957-5388,thewhites@houston.rr.com
}

\begin{abstract}
The canonical form of the Alcubierre warp drive metric is considered to gain insight into the mathematical mechanism triggering the effect. A parallel with the Chung-Freese spacetime metric is drawn to demonstrate that the spacetime expansion boost can be considered a $3+1$ on-brane simplification for higher dimensional geometric effects. The implications for baryonic matter of higher dimensional spacetime, in conjunction with the Alcubierre metric, are used to illustrate an equation of state for dark energy. Finally, this combined model will then be used to outline a theoretical framework for negative pressure (an alternative to negative energy) and a conceptual lab experiment is described.
\end{abstract}

Keywords: Warp Drive, General Relativity, Extra Space Dimensions, Dark Energy, Cosmology.

PACS: 04.20.Gz, 04.50.+h, 11.25.-w, 98.80.-k, 98.80.Qc.

\section{INTRODUCTION}

What if it were possible to cover vast interstellar distances in arbitrary short periods of time? What if one could fabricate an Deus ex Machina that could be employed to accomplish such a goal? What would this machine look like? How would an engineer or physicist go about the process of fabricating such an apparatus? All daunting questions to be certain, but there are some potential answers - at least theoretical ones anyway.

The notion of inflation is fairly well known in the literature and has some common acceptance in its most current form evolved from Guth's original model. It is this mechanism that motivated Alcubierre (1994) to construct a metric that employed inflation to allow an observer to traverse vast distances in arbitrary times by riding inside a 'warp sphere.' The benefit of employing inflation is that one never violates the speed of light locally, one merely rides space itself to achieve the desired result of getting to Alpha Centauri in less than four Earth years. Now time for some bad news. The Alcubierre model requires negative energy, which is obviously not your typical gardenvariety matter that you can pick up at the grocery store in the boson/lepton aisle. It is this requirement that relegates Alcubierre's model, in its original form, to simply being a toy mathematical construct.

All hope need not be lost - yet. The principal author has done some follow-on work with the original metric to put it into canonical form (White 2003). In this form, it is easier to see what may really be responsible for the space expansion/contraction. In the metric's canonical form, to be discussed later in this paper, one can see that the boost is the driving phenomenon acting on an initial velocity. The space expansion/contraction is a dependent effect, sort of like a space drag around the traveling warp sphere space. That still leaves the negative energy issue to be more formally addressed. It is this issue that will be tackled in this paper by means of comparison with higher dimensional metrics and the canonical Alcubierre metric.

CP813, Space Technology and Applications International Forum-STAIF 2006, edited by M. S. El-Genk (C) 2006 American Institute of Physics 0-7354-0305-8/06/\$23.00 


\section{INFLATION: THE ALCUBIERRE WARP DRIVE MODEL}

Alcubierre (1994) derived a metric motivated by cosmological inflation that would allow arbitrarily short travel times between two distant points in space. The "warp drive" spacetime uses coordinates $(t, x, y, z)$ and curve (or worldline) $\mathrm{x}=\mathrm{x}_{\mathrm{s}}(\mathrm{t}), \mathrm{y}=0, \mathrm{z}=0$, lying in the $\mathrm{t}$ - $\mathrm{x}$ plane passing through the origin. Note that $\mathrm{x}_{\mathrm{s}}$ is the $\mathrm{x}$-axis coordinate position of the moving spaceship frame. The metric specifying this spacetime is (Alcubierre, 1994):

$$
d s^{2}=-c^{2} d t^{2}+\left[d x-v_{s}(t) f\left(r_{s}\right) d t\right]^{2}+d y^{2}+d z^{2},
$$

where $v_{s}(t) \equiv d x_{s}(t) / d t$ is the velocity associated with the curve, $r_{s} \equiv\left[\left(x-x_{s}(t)\right)^{2}+y^{2}+z^{2}\right]$ is the Euclidean distance from the curve, and $\mathrm{c}$ is the speed of light. (Note: all physical quantities are assumed to be in MKS units unless otherwise specified.) The "top hat" shaping function $f\left(r_{s}\right)$ is any smooth positive function that satisfies $f(0)=1$ and decreases away from the origin to vanish when $r_{s}>R$ for some $R$. The geometry of each spatial slice is flat, and spacetime is flat where $f\left(r_{s}\right)$ vanishes but is curved where it does not vanish.

The driving phenomenon of this metric is postulated to be the York extrinsic time, $\theta$. This quantity is defined as (Alcubierre, 1994):

$$
\theta=\frac{v_{s}}{c} \frac{x_{s}}{r_{s}} \frac{d f}{d r_{s}}
$$

The York extrinsic time behavior of the warp drive metric provides for the simultaneous expansion of space behind the spacecraft and a corresponding contraction of space in front of the spacecraft (see Figure 1 below). Thus a spacecraft can be made to exhibit an arbitrarily large apparent faster-than-light (FTL) speed $\left(\mathrm{v}_{\mathrm{s}}>>\mathrm{c}\right)$ as viewed by external coordinate observers.

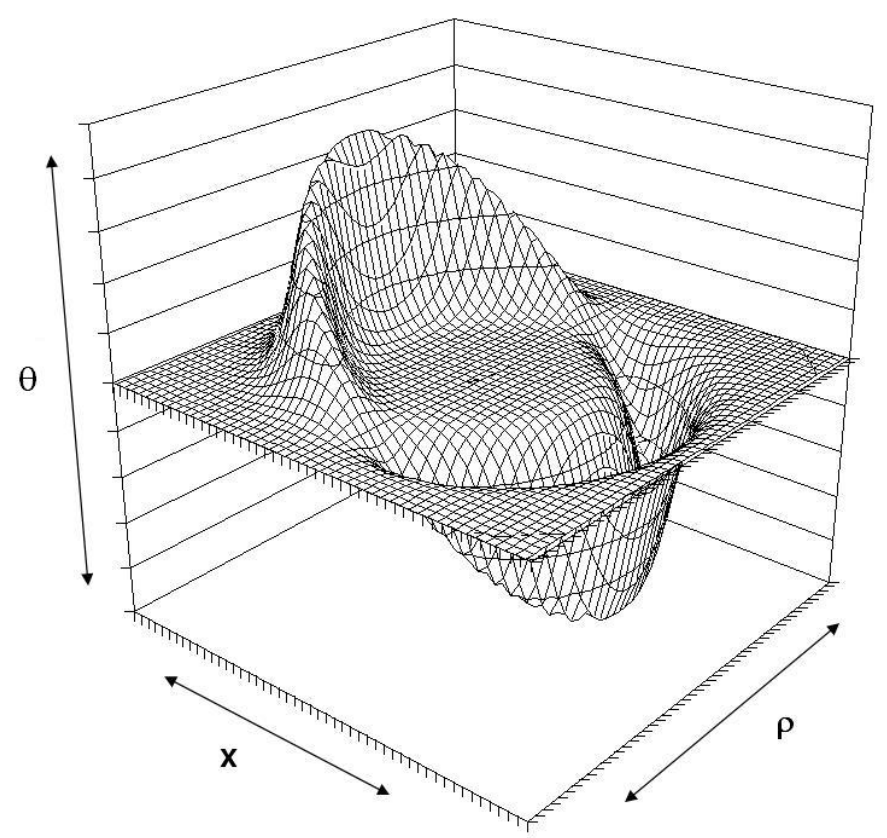

FIGURE 1. York Extrinsic Time $(\theta)$ Plot.

This idea is intended as an applied extension of the hypothesis that the early universe underwent a rapid inflationary expansion phase immediately after the onset of the Big Bang, and the notion that the York extrinsic time is the driving mechanism behind the metric is a reasonable assumption. However, as proven in White (2003) this leads to 
an unusual symmetry of the energy density derived from Einstein's General Relativity field equations (Alcubierre, 1994):

$$
T^{00}=-\left(c^{4} / 8 \pi G\right)\left[v_{s}^{2}(t) \rho^{2} / 4 r_{s}^{2} c^{2}\right]\left(d f / d r_{s}\right)^{2},
$$

where $G$ is the Universal Gravitation Constant and $\rho=\left(y^{2}+z^{2}\right)^{1 / 2}$. The symmetry results in a scenario where the choice of positive x-axis (the axis of the above energy density toroid) is arbitrary. In other words, the ship does not know which direction to go - forward or backward. The symmetry paradox can be resolved by putting the Alcubierre warp drive metric into its canonical form using Rindler's method. For readers not familiar with the canonical form of a spacetime metric, consider the Schwarzschild metric as an academic example. The advantage of the canonical form is that one can extract the potential, $\phi$. Using the potential one can then extract the field equation for the spacetime expansion boost, $\gamma$ (White, 2003):

$$
\gamma=\cosh \left[0.5\left(\ln \left|1-\left(v_{s} / c\right)^{2} f^{2}\left(r_{s}\right)\right|\right)\right],
$$

Figure 2 shows a cross-section of the boost topology throughout the sphere. There is a strong boost gradient across the horizon of the sphere settling to a flat region representing the inside of the sphere.

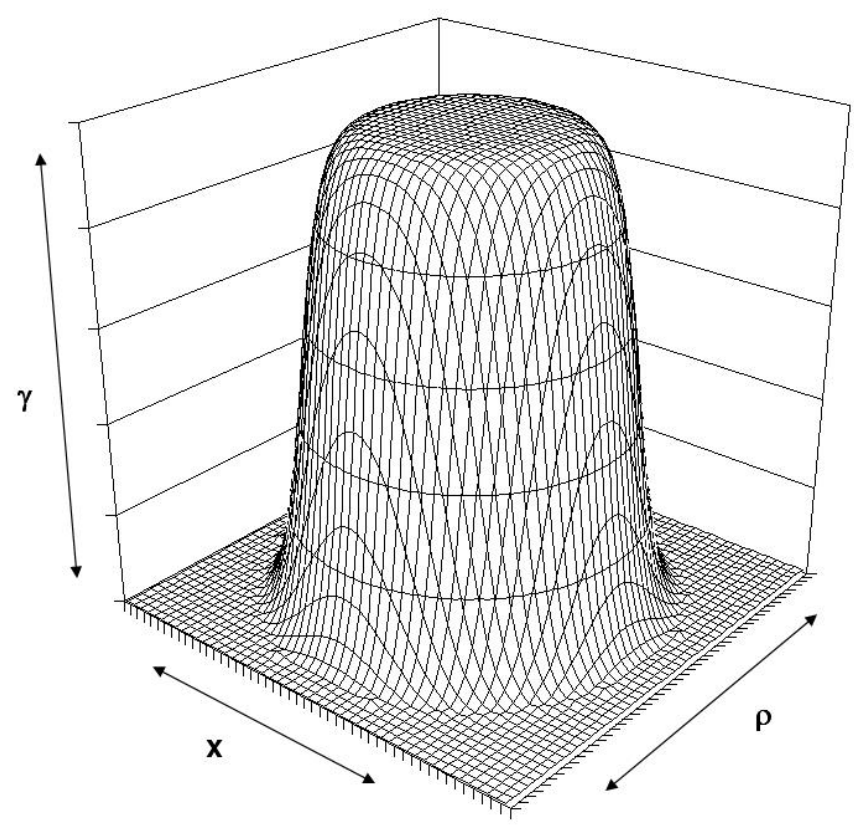

FIGURE 2. Spacetime Expansion Boost $(\gamma)$ Plot.

So what is the significance of the spacetime expansion boost? Simply put, the boost can be thought of as a scalar multiplier acting on an initial velocity $\left(\mathrm{v}_{\text {ini }}\right): \mathrm{v}_{\mathrm{s}} \approx \gamma \cdot \mathrm{v}_{\text {ini }}$. The expansion and contraction of space are a secondary effect and might be considered an analog of pressure gradients around a moving sphere in a fluid. This aspect of the metric is more fully explored in White (2003). The scalar characteristic of the boost can be demonstrated by considering the null geodesics for, say $\mathrm{v}_{\mathrm{s}}=10 \mathrm{c}$, within the region of the warp field. Even though the worldlines in the warp field region are spacelike for external observers, the moving frame itself never travels outside its local comoving light cone and thus does not violate Special Relativity (see Figure 3). 


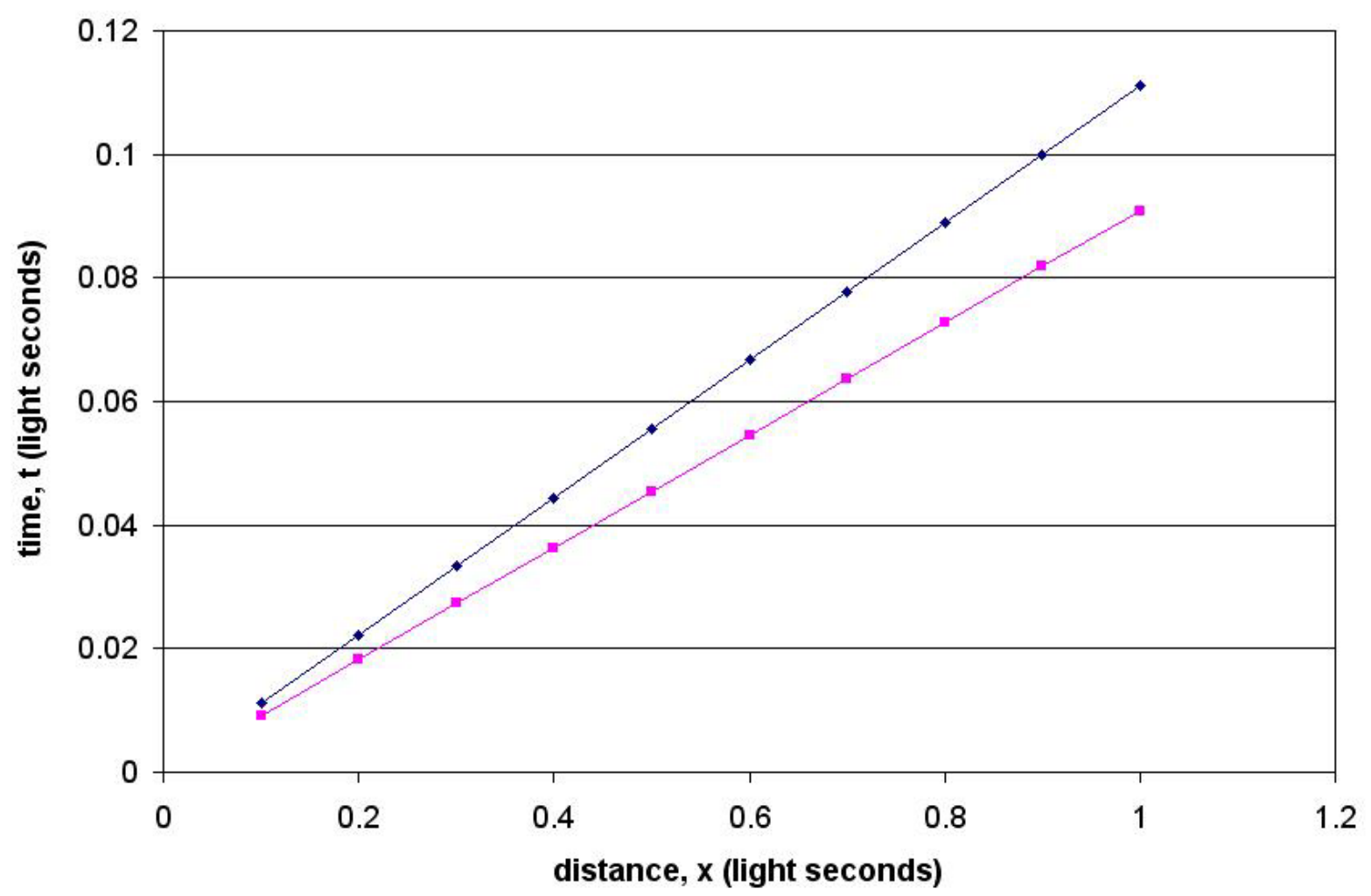

FIGURE 3. Photon Worldlines Inside the Warp Field Region.

\section{BRANE WORLD: INTRODUCTION TO THE CHUNG-FREESE MODEL}

We now consider an instance of a higher dimensional spacetime model. Chung and Freese (2000) derived and explored a metric representing a higher dimensional universe that includes our own $3+1$ spacetime dimensions. In this particular model, our universe exists on a "brane" and the space spanned by the extra space dimensions is called the "bulk." Each of the $n$ extra space dimensions is of finite extent $\approx 2 \times 10^{(32 / n)-17}$ centimeters (Davis, 2004). The number of extra space dimensions could be argued, but the discussion will be restricted to the original metric format. The metric representing this model is (Chung and Freese, 2000):

$$
d s^{2}=-c^{2} d t^{2}+\frac{a^{2}(t)}{e^{2 \mathrm{kU}}} d X^{2}+d U^{2}
$$

Equation (5) is a modified Friedmann-Robertson-Walker (FRW) metric where the $\mathrm{dX}^{2}$ term represents our normal affine space (on the brane), the $\mathrm{dU}^{2}$ term represents the bulk with our brane being located at $\mathrm{U}=0$, $\mathrm{a}(\mathrm{t})$ is the typical cosmological expansion parameter (or scale factor), and $\mathrm{k}$ is the compactification factor of the extra space dimensions which is assumed to be one (this choice is arbitrary). As a mental construct to aid in visualization, consider a 2-dimensional (2D) affine surface and label it as a brane upon which 2D Earthlings live their 2D lives. In this scenario, the $\mathrm{x}$ - and $\mathrm{y}$-axes make up the $\mathrm{x}$-y plane which will be labelled the brane. Thus $\mathrm{dx}^{2}+\mathrm{dy}^{2}$ would be the $\mathrm{dX}^{2}$ in (5). The remaining $\mathrm{z}$-axis would be the axis pointing normal to the $\mathrm{x}$-y plane (or brane) and hence into the bulk. Here, $\mathrm{dz}^{2}$ would be the equivalent to $\mathrm{dU}^{2}$ in (5). To say that one has a non-zero off-brane bulk coordinate in this case would simply mean that the z-coordinate would be non-zero.

As was done earlier, considering the null geodesic solutions $\left(\mathrm{ds}^{2}=0\right)$ allows us to derive the following relationship for photon worldlines in this model: 


$$
\frac{d X}{d t}=\frac{c e^{\mathrm{kU}}}{a(t)} \sqrt{1-\frac{d U^{2}}{c^{2} d t^{2}}}
$$

Note that for large off-brane bulk coordinates ( $\mathrm{U}>>1$ ), $\mathrm{dX} / \mathrm{dt}$ can be made to be arbitrarily large. If plotted, these worldlines would be spacelike as previously shown for the Alcubierre warp drive metric in Figure 3.

\section{NULL GEODESIC SOLUTIONS: A COMPARISON BETWEEN MODELS}

We now draw some parallels between mathematical models by means of their null geodesics. With both models, the light rays could be made to have a spacelike appearance. In the Alcubierre model the spacetime expansion boost was conjectured to be the driving phenomenon, while in the Chung-Freese model the off-brane bulk location served this role. Conceptually, perhaps we can thus state that the boost of a $3+1$ spacetime model is a scalar correction factor for higher dimensional geometric effects here on the brane. Or more simply put:

$$
\gamma \approx e^{U} .
$$

As the boost $\gamma$ gets larger, there is a corresponding shift further off-brane for the local inertial frame (LIF) experiencing that boost. We have now drawn an interesting parallel between the two models suggesting that a high spacetime expansion boost is a scalar simplification of higher dimensional geometric effects here on the brane. What would be the physical implications of this? Consider a particle accelerator. As an electron attains an arbitrarily high spacetime expansion boost relative to a laboratory observer, the electron is actually obtaining a nonzero bulk coordinate U. As it leaves the $3+1$ brane (obtains high boost value), its ability to interact electromagnetically with our brane is diminished.

We will invoke the 2D example again to illustrate this point. Say that the 2D laboratory is located on the $x-y$ plane at $\mathrm{z}=0$ which we will label as being on the brane. The $2 \mathrm{D}$ electron in the particle accelerator obtains a larger nonzero $\mathrm{z}$ value (off-brane) as its boost gets larger. Unless a photon is at the same $\mathrm{x}, \mathrm{y}, \mathrm{t}$, and $\mathrm{z}$ coordinate, it cannot interact with the electron. In this example, the $2 \mathrm{D}$ lab must be able to generate photons at the same non-zero $\mathrm{z}$ value as the electron in order to continue to interact with it.

\section{INFLATION AND THERMODYNAMICS}

Now let us again consider the Alcubierre model. Specifically, let us consider the spherical region (a.k.a. warp field region) of constant spacetime expansion boost. Even though a toroidal energy density brings about the spherical region, the sphere has a uniform boost within and hence a uniform potential to an outside observer. So the Alcubierre model's spacetime expansion boost exhibits mathematical characteristics similar to that of a Gaussian spherical surface held at a constant electrostatic potential. To an outside observer, the Alcubierre warp field sphere has a uniform energy density.

The next step is to consider what happens when expanding the spherical warp region while maintaining the same relative boost value for the Gaussian surface. We will limit our discussion to the $3+1$ brane and employ traditional thermodynamic relations to consider the scenario. From the First Law of Thermodynamics, with no heat flow, we have the following:

$$
d E=-p_{s} d V .
$$

The term $\mathrm{dE}$ can be replaced by $\rho_{\mathrm{s}} \mathrm{dV}$, which is the total energy for the warp sphere with the same volume change $\mathrm{dV}$ as on the right side of (8). This leads to the equation of state relating the pressure of the warp sphere, $\mathrm{p}_{\mathrm{s}}$, to its energy density, $\rho_{\mathrm{s}}$ :

$$
\rho_{s}=-p_{s} .
$$


This provides us with the insight that if $\rho_{\mathrm{s}}$ of the original warp sphere is negative, then the corresponding $\mathrm{p}_{\mathrm{s}}$ is positive. However, (9) shows that the converse is true, $\rho_{\mathrm{s}}$ is positive and so $\mathrm{p}_{\mathrm{s}}$ is negative. Recall that the spacetime expansion boost for the Alcubierre model could be made to be arbitrarily high depending on the choice of input variables. A high boost is clearly not an exclusive feature common only to negative energy densities and can be readily obtained in the lab provided powerful enough equipment. It should be further noted that (9) is the equation of state for the cosmological vacuum energy, and it is also the (astronomically measured) equation of state for dark energy.

\section{DARK ENERGY AND PROPOSED EXPERIMENT}

So what if, then, we indeed live in a higher dimensional universe other than the traditionally accepted $3+1$ spacetime dimensions (on the brane), and equation (7) makes certain predictions about its behavior? Let us reconsider what happens as a LIF assumes a large boost with respect to a coordinate observer here on the brane (recall the earlier discussion). This exercise will again be restricted to considering what happens within a warp field as defined by the Alcubierre metric.

As the Alcubierre LIF (ALIF) warp sphere takes on a large spacetime expansion boost and starts to move off-brane as suggested by equation (7), the photons emitted from it will not be able to intersect the coordinate observer (lab) frame because the photons were emitted off-brane from the ALIF. This is the reverse scenario we discussed earlier where photons on the brane were not able to interact with an electron off of the brane. Unless something acts on the off-brane photons to reduce their boost, they will not be detected by the on-brane coordinate observer. Hence, an Earthbound coordinate observer will most likely see a dimming effect when viewing the ALIF as its spacetime expansion boost increases. Similarly, the ALIF will see his/her surrounding universe grow dimmer and dimmer as photons emitted on-brane cannot reach the now off-brane ALIF.

It is this inability to electromagnetically interact that we want to focus our attention on. In the previous section we made note of the fact that a positive energy density was commensurate with a negative pressure for the warp sphere. Equation (7) suggests that all matter within that sphere cannot electromagnetically communicate with matter here on the brane. However, we can still detect its pressure and energy density effects. These are characteristics familiar to another form of energy in the cosmos, called "dark energy." Dark energy can be mathematically modeled the same way that we have just described above.

Therefore, we have sketched a theoretical framework whereby baryonic matter can - at least mathematically - be made to adopt similar characteristics to dark energy including its inability to electromagnetically interact. It is interesting that this line of thought has its roots in cosmological inflation. In this process, we noted indirectly that a toroidal positive energy density could yield a spherical negative pressure region. Perhaps the reader is still struggling with the concept of how a toroidal positive energy density develops a spherical region of negative pressure. The short answer is that is what the math predicts. The intuitive answer is that it is a consequence of living in a higher dimensional universe and having the requirement of satisfying the First Law of Thermodynamics.

Since we know how to make a large spacetime expansion boost value, a test configuration could be invoked conceptually as shown in Figure 4. The figure depicts a modified Michelson-Morley Interferometer setup that makes use of a $1 \mathrm{~cm}$ diameter toroidal-ring of positive energy density on one leg of the interferometer. A He-Ne laser beam $(\lambda=633 \mathrm{~nm})$ is split allowing one part of the beam to pass through the center of the ring and hence the spherical warp field region. This warp field region will induce a relative phase shift between the split beams that could be detectable provided the magnitude of the phase shift is sufficient. If the desired phase shift goal were set to be roughly $1 / 4^{\text {th }}$ wavelength (reasonable expectation), then the necessary boost field is on the order of 1.0000001 to 1.0000002 . From a purely Special Relativistic perspective, this equates to a velocity of $\sim 0.0004 \mathrm{c}$ which could be achieved potentially with a toroidal ring of plasma. Additionally, we could take the route of acting on the boost by means of the potential or gauge, $\gamma=\cosh (\phi)$. In this scenario, we would employ a ring of capacitors driven at high voltage and moderately high frequencies to act on the potential $(\phi)$ of the ions within the dielectric. Work on this front is currently ongoing to develop system specific parameters such as RF amplifier power requirements, capacitor size, tank circuit details, and more. 


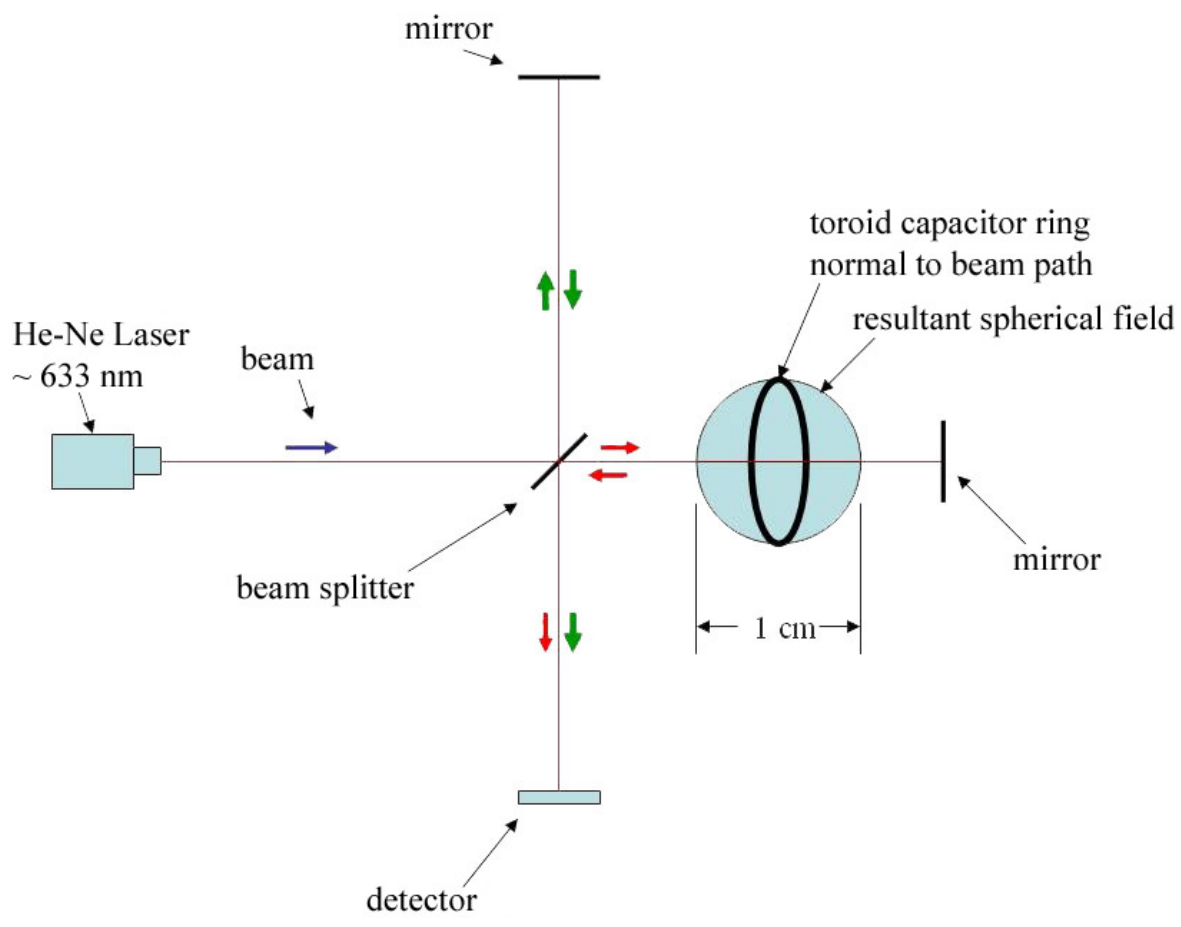

FIGURE 4. Proposed Lab Experiment.

\section{CONCLUSION}

We used Rindler's method to extract the canonical form of the Alcubierre warp drive metric in order to properly pose this FTL geometry into the Chung-Freese modified FRW metric for cosmological inflation in higher dimensional spacetime. This led to the remarkable discovery that a spacecraft's spacetime expansion boost in the Alcubierre warp spacetime actually represents a movement of the spacecraft off our local brane $(3+1$ dimensional spacetime) and into the higher dimensional bulk space. The Alcubierre warp spacetime expansion boost merely acts as a scalar multiplier acting on an initial velocity. The consequence of this is that the equation of state for the energy density and pressure that induces this effect is equivalent to the dark energy equation of state and the equation of state for the vacuum energy in space. This suggests a conceptual laboratory experiment whereby a toroidal positive energy density induces a negative pressure warp field.

\section{ACKNOWLEDGMENTS}

H. G. White would like to thank J. Woodward, P. March, D. Fletcher, E. Davis, and A. White for earnest discussions that facilitated the development of the presented work. E. W. Davis would like to thank the Institute for Advanced Studies at Austin for supporting this work.

\section{REFERENCES}

Alcubierre, M., "The warp drive: hyper-fast travel within general relativity," Class. Quant. Grav. 11, L73-L77 (1994).

Chung, D. J. H., and Freese, K., "Can geodesics in extra dimensions solve the cosmological horizon problem?,” Phys. Rev. D 62, 063513 (2000). 
Davis, E. W., Teleportation Physics Study, Final Report AFRL-PR-ED-TR-2003-0034, Air Force Research Laboratory, Air Force Materiel Command, Edwards AFB, CA, 2004, pp. 50-51.

White, H., “A Discussion on space-time metric engineering," Gen. Rel. Grav. 35, 2025-2033 (2003). 